\title{
Design of Temperature and Humidity Automatic Alarm Recorder Upper Machine Based on Delphi
}

\author{
Baohua Jiang ${ }^{\mathrm{a}}$, Peixue Liu ${ }^{\mathrm{b}^{*}}$, Yujie $\mathrm{Chen}^{\mathrm{c}}$ and Xiaoxiao Shi ${ }^{\mathrm{d}}$ \\ Qingdao Huanghai University Mechanical and Electrical Engineering College,China

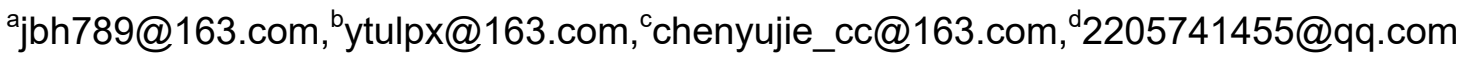

Keywords: Temperature and humidity;Delphi7.0;PC; Recorder

\begin{abstract}
Because of the low efficiency of manual operation of the traditional temperature and humidity record, a kind of temperature and humidity automatic alarm recorder upper machine system is designed using the computer processing technology, the RS485 communication technology and the human-computer interaction technology. And Delphi 7.0 language was used in development of the data management system. Test results show that the software meets the demand of practical application, reasonable design, system operation is stable and reliable.
\end{abstract}

\section{Introduction}

China's current rapid development of science and technology, in real life people keep information his way of life, so the requirement of production and living are increasingly demanding. At present, the Chinese mainland for agricultural environment parameters for processing system design of the intelligent level is not high, the industrial environment parameters monitoring is also facing many problems, such as inspection site condition is complex, complex and not easy to send unattended, so artificial meter reading this kind of work mode under this conditions is extremely inconvenient and inefficient execution. So there is an urgent need to a remote control, real-time monitoring, economic and efficient data acquisition system.

This topic design automatic alarm - temperature and humidity recorder PC, with functions of real-time display (temperature and humidity parameter data, real-time display) after corresponding treatment, transfinite alarm function (upper and lower limit alarm point users to set temperature and humidity, terminal data more than limit alarm events), data query function (convenient comparative analysis), the user management functions (user can increase and decrease operation, modify the account and password), remote communication function (in the remote monitoring and control).Through the Delphi software programming realization, has features of simple structure, high reliability, low cost, can be achieved at any time, anywhere the acquisition of data, data analysis and processing, a machine under remote control terminal, has the very broad market application value.

\section{The System Overall Design}

The PC system of temperature and humidity automatic alarm recorder application development design using the programming tool Delphi7 development platform, system mainly consists of five main modules, they respectively are: the operator management, the main page, basic operation, basic information and information about the module (Fig. 1). 


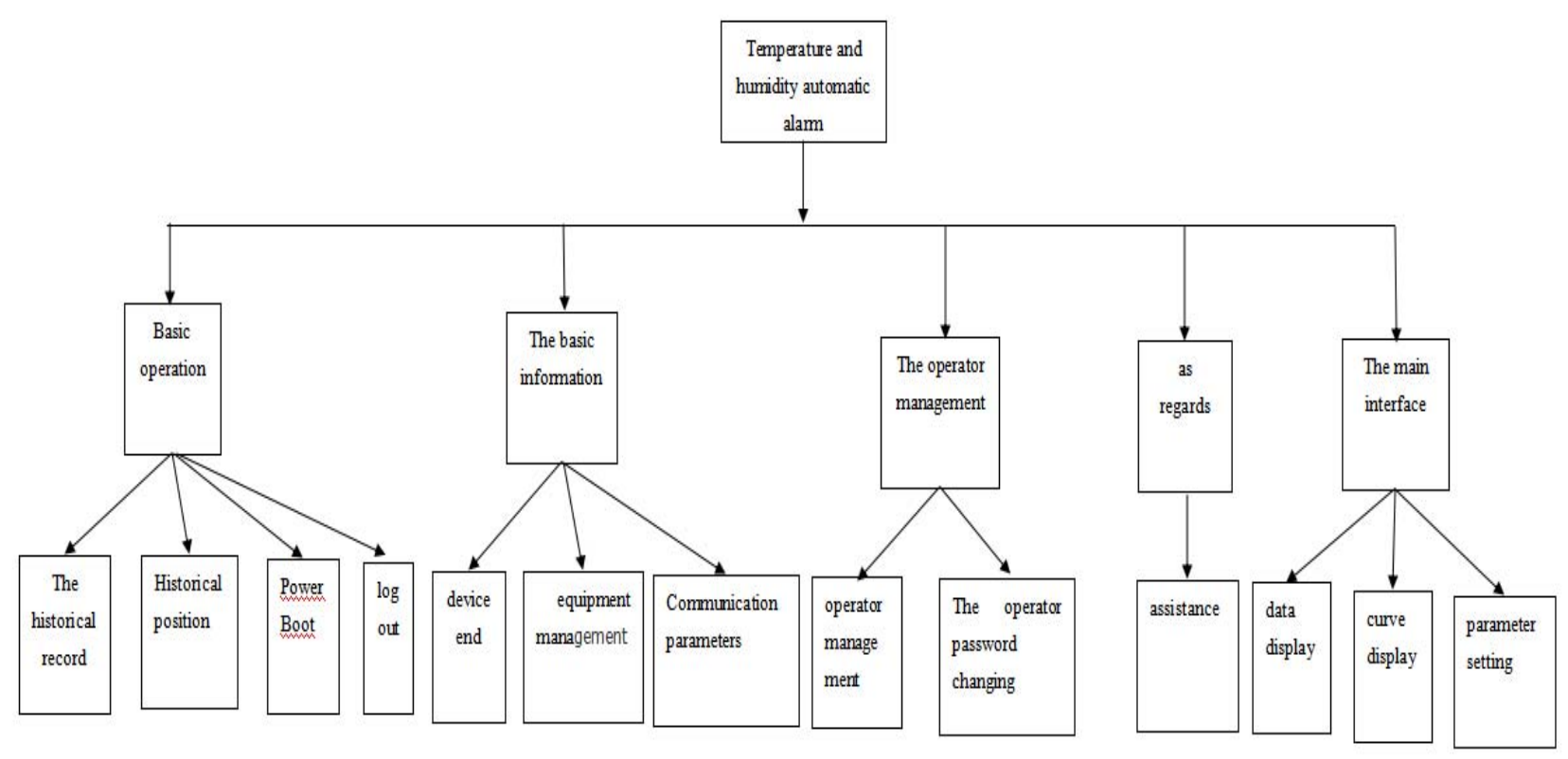

Fig.1 Function system diagram

\section{The development environment}

The advantage of Delphi: Delphi and object-oriented method based on the form, huge database support, extremely fast compilers, component technology and the close integration of Windows programming features is to make developers choose the original, and Delphi powerful, easy to use, simple and efficient, beautiful code, compilation speed, has the support of components and rich learning resources, fully supports Pointers, support object-oriented, inline assembler, for rapid development, database development, system level and very convenient hardware control programming [1].

Delphi 7 improvement and fixed a new technique to make it more robust (SOAP support and Data - the emergence of the Snap), provide for more new technology (such as Windows XP themes or UDDI) support.

Access database: Microsoft Office Access is the Microsoft database engine graphical user interface and software development tools together a database management system. Microsoft Office Access has simple storage mode, object oriented, friendly interface, easy operation, etc, and the Access is a visualization tool, and the Windows have exactly the same style, the user can use the mouse to drag and can generate very easy and intuitive to objects and applications. Wizard, the system also provides a database table generator, query wizard, query builder, form wizard and table wizard, report designer, report the wizard tool such as, so simple operation, easy to use and control.

\section{Software design and test}

In view of the traditional temperature and humidity record low efficiency of manual operation, work, combined with the computer processing technology, the RS485 communication technology, the human-computer interaction technology, put forward a kind of temperature and humidity automatic alarm recorder upper machine system design. The system has realized the real-time 
monitoring of temperature and humidity data, processing, transmission, and the function of human-computer interaction, the whole system USES modular design, simple and convenient structure design, function perfect, systematic upgrading, improve the efficiency of the workers, reduces the cost, will significantly improve the traditional temperature and humidity data processing work. Let go at the same time, along with the computer technology, using the hoisting system of the computer technology will be more convenient work rate, the system will be more widely used.

Comprehensive in the information age today, the arrival of intelligent temperature and humidity measurement technology is rapid development vigor, there are reasons to expect that the development of the technology and mature will bring to people's production and living is extremely far-reaching influence.

The user login:Main complete access database connection and user login user name login information verification.Using ADO controls TADOConnection and connect ACCESS database, called kdl380 connection code

Adoconnection1. The connectionstring: $=$ '. The Provider $=$ Microsoft Jet. The OLEDB. 4.0;JetOLEDB: DatabasePassword = golddancer; The DataSource = '+ extractfilepath (paramstr (0)) +' kd1380. MDB.Persist Security Info = True ';

Connection is successful after all the user name will be added to the ComboBox1 control list, for the first time to use the software only one administrator user wen-xiu xu, initial password is administrator username xuwenxiu hanyu pinyin mode, the operator name and fill in the characters in the operator password, click "ok" button control to trigger controls the OnClick event, the system will look for in the database list of links to the corresponding characters, if does not have a corresponding character in the database, the interface will show "sorry, wrong password!", if the input the correct account Numbers and passwords, will withdraw this landing interface, enter the main interface.

Display interface:Through the menu in the main page controls TmainMenu can be very convenient to design the main menu.Through the control of the Items property editor for the design of the various functions of menu.StringGrid component in the component displays a string of grid work, basic resemblance in electronic form.It by using the string in the grid and related objects, simplify the operation of the system user.StringGrid component provides many properties, such as the concept of control network especially attributes, using tables are also included in this framework and methods to the operation of the incident response system users.StringGrid is the function of each string in the grid with object, the user use the string, the string containing the behavior of the instruction and information were encapsulated by these objects.String - the Grid control is used to convenient equipment of various information list.

The system test:Using virtual serial debugging software (see Fig. 2) virtual configuration a communication port on the computer, virtual port can communicate with each other.Using the serial debugging assistants to simulate a machine using virtual out port to communicate with the system test.

Open the serial debugging assistants and the system software, select the set attached to a port, baud rate and modify the right parameter. 


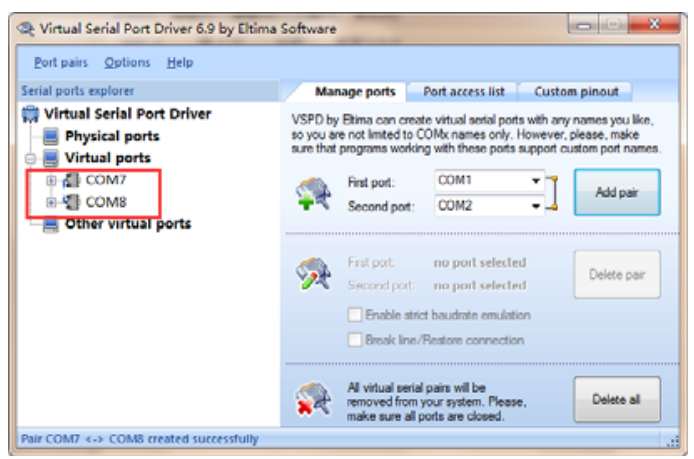

Fig.2 VSPD virtual serial port software

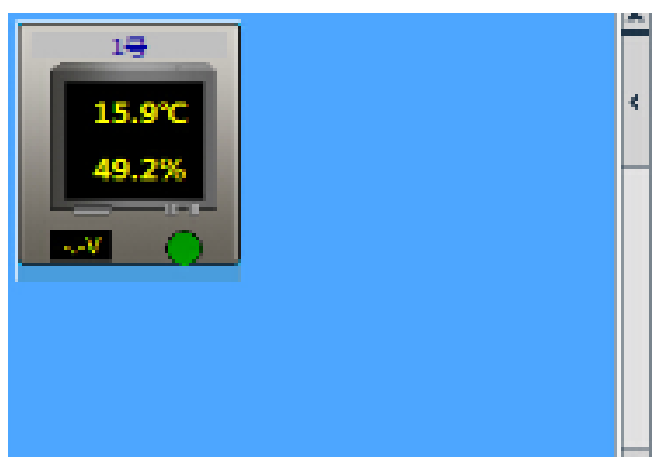

Fig.3 Test results

From the point of the test way, on the one hand, the PC as the receiver, can receive data accord with standard of RS485 communication protocol, and can according to the set of data conversion model will be the original data in the corresponding position of PC display correctly. PC as command, on the other hand, the sender, the emulated serial assistant interface, we can clearly see the data according to the standard of RS485 communication protocol model was translated into the correct order information HEX code. This suggests that the upper machine communication function can stable (Fig. 3).

\section{Conclusions}

Given the Delphi 7.0 write the potential advantages of PC system, put forward by Delphi 7.0 write the implementation scheme of PC system management data, analysis of the test results through the system test, the system working in good condition, can work stably for a long time, can achieve the required basic level, for the system of social marketing has laid a good foundation.

\section{Acknowledgments}

During the process of this paper, the relevant teachers give lots of help and support. This topic is higher school science and technology plan projects subject of Shan Dong Education Ministry -The Design of Cloud Platform for Internet of Things Environment Based on B/SandThe Design of Internet of things remote identification and information collection system based on 4G(J15LN59) .

\section{Reference}

[1] Han-liang li. Temperature and humidity based on Delphi system development example is pithy [M]. Beijing: people's posts and telecommunications publishing house, 2009.

[2] KuiChun, Chen Zigong. Temperature and humidity monitoring system based on Delphi database development instance (fifth edition) [M]. Beijing: mechanical industry publishing house, 2013.

[3] S.Q.X, ie Y.L.T u.A WWW - -based Information Management System for Rapid and Integrated Mould Product Development[J]. International Journal of Advanced Manufacturing Technology, 2011 (3) : 32-45.

[4] Pan Wenlin.Database application system product development practice[M]. Beijing: mechanical industry publishing house, 2010.

[5] Sun Zuliu. The power of the 21st century scientific and technological progress[M]. Beijing: weapon industry press, 2012. 